\title{
Prevalence and antibiotic susceptibility pattern of Coagulase Negative Staphylococcus (CoNS) in neonatal septicemia
}

\author{
Vijetha Sajjanar', D. E. Premalatha, ${ }^{2, *}$ \\ ${ }^{1}$ PG Student, ${ }^{2}$ Assistant Professor, Dept. of Microbiology, Rajiv Gandhi University of Health Sciences, Shimoga, Karnataka, \\ India
}

*Corresponding Author:

Email: deprema@hotmail.com

\begin{abstract}
Introduction: Neonatal septicemia is important cause of neonatal mortality and morbidity. Neonatal Coagulase negative staphylococci(CoNS) infections are rarely fatal, but they cause significant morbidity, especially among very low birth weight infants.

Objectives of the study: 1 . To know the prevalence of CoNS in neonatal sepsis; 2 To determine the antibiotic susceptibility pattern of CoNS in neonatal sepsis.

Materials and Methods: A prospective study was conducted on blood samples of suspected neonatal septicemia between July 2017 and December 2017 received at Department of Microbiology, SIMS, Shimoga. Under aseptic precautions samples were collected and inoculated into BHI broth and subcultures were performed on MacConkey agar and blood agar plates and growth was processed by standard bacteriological technique. Antibiotic susceptibility testing was performed by Kirby Bauer Disk Diffusion test. Results were interpreted as per CLSI guidelines.

Results: Out of 156 samples 98 were culture positive and 58 were culture negative. Most common organism isolated was Coagulase negative staphylococci(CoNS) 48.97\%(48/98),Klebsiella species21.4\%(21/98),Staphylococcus aureus10.2\%(10/98),Gram Negative non fermenters 9.1\%(9/98),Escherichia coli 2.04\%(2/98), Citrobacter spp 4.08\%(4/98),Pseudomonas spp 1.02\%(1/98),Enterococcus spp 1.02\%(1/98),Streptococcus spp 1.02\%(1/98),Candida spp $1.02 \%(1 / 98)$. Gram positive organisms were highly sensitive for Linezolid, Vancomycin moderately sensitive for Gentamicin, Cotrimoxazole, ciprofloxacin and least sensitive for Erythromycin, Clindamycin, ciprofloxacin, Azithromycin, Cefoxitin(MRCoNS)

Conclusion: This study showed emergence of Coagulase negative staphylococci(CoNS) as the most common pathogen in neonatal septicemia. Prevalence of MRCoNS and multidrug resistance among Coagulase negative staphylococci(CoNS) is seen.
\end{abstract}

Keywords: Coagulase negative staphylococci, Neonatal septicemia, MRCoNS, Antibiotic susceptibility.

\section{Introduction}

Neonatal septicemia is the most important cause of neonatal mortality and morbidity. Neonatal mortality rate is one of the indicators of health status of nation. It is major public health problem in developing countries. Neonatal septicemia refers to systemic bacterial infection with positive blood culture in the first 4 weeks of birth. ${ }^{1}$ Two types based on the age of onset: Early onset septicemia $(<72 \mathrm{hrs})$ and late- onset septicemia [LOS] ( $\geq 72$ h 28 days).Risk factors for EOS are Preterm premature rupture of membranes, maternal fever, chorioamnionitis, foul smelling liquor, urinary tract infection, multiple vaginal examinations. Risk factors for LOS are Preterm, low birth weight, formula feeding, invasive procedures, overcrowding, understaffing, lack of asepsis. Common organism causing neonatal sepsis are Klebsiella pneumoniae, Staphylococcus aureus, Coagulase negative staphylococci(CoNS),Escherichia coli, Pseudomonas species, Citrobacter species, Acinetobacter species, Streptococcus species. ${ }^{2}$ The isolation of Gram- positive organisms has increased significantly compared to gram negative organisms. CoNS is usually considered commensal but when two positive blood cultures are obtained it is pathogenic isolate according to CDC criteria. Coagulase-negative staphylococci (CoNS) are the most prevalent pathogens causing late onset sepsis in neonates. Neonates become colonized by microorganisms present in environment within first week of life. This causes the risk of CoNS infection to increase with use of Central venous catheter, parentral nutrition, mechanical ventilation and with exposure to other invasive skin or mucosa breaking procedures. ${ }^{3,4}$ Neonatal CoNS infections are rarely fatal, but they cause significant morbidity, especially among very low birth weight infants. In addition, CoNS frequently display multiresistance to antibiotics. Judicious use of antibiotics, aggressive supportive care, proper hygiene methods can prevent neonatal mortality. Neonatal sepsis causative bacteria and pattern of susceptibility to antibiotic by organisms are different in each hospital and region. ${ }^{5}$ Therefore the present study was undertaken to know the prevalence and antibiotic susceptibility of Coagulase negative staphylococci in neonatal septicemia patients at a tertiary care hospital, SIMS, Shimoga.

\section{Materials and Methods}

The study was carried out in Department of microbiology, attached to McGann teaching hospital, SIMS, Shimoga. A prospective study was conducted during the period July 2017 and December 2017 at 
Department of Microbiology, SIMS, Shimoga. A total of 156 blood samples were collected from all clinically suspected neonatal sepsis patients.

Isolation and Identification: Under aseptic precautions about $1 \mathrm{ml}$ of blood was drawn and inoculated into BHI(brain heart infusion broth) and incubated at $37^{\circ} \mathrm{C}$. The samples were processed by standard bacteriological procedure. ${ }^{6}$ Subcultures were done blindly or on earliest visual detection of turbidity on days 1, 4 and 7 on MacConkey agar and blood agar plates and incubated at $37^{\circ} \mathrm{c}$ aerobically for $24 \mathrm{hrs}$. Absence of microbial growth after 7 days were given as culture negative. The organisms isolated were identified by colony morphology, Gram stain, relevant standard biochemical methods. Catalase and oxidase test was done. Members of the family enterobacteriaceae were identified by indole production, $\mathrm{H}_{2} \mathrm{~S}$ production, citrate utilization and other tests. Gram positive organisms were tested for Coagulase test, catalase test, bacitracin sensitivity and optochin sensitivity. Cultures that yielded commensal species (CoNS) were reviewed using CDC criteria for CoNS related blood stream infection where minimum of two positive blood cultures are required to fulfill the criteria. ${ }^{7,8}$

Antimicrobial susceptibility testing: Antimicrobial susceptibility testing was carried out on Mueller-Hinton agar by Kirby Bauer Disk Diffusion method. Following antibiotics were used for gram positive organism are Linezolid $(10 \mu \mathrm{g})$, Vancomycin $(30 \mu \mathrm{g})$, erythromycin(15 $\mu \mathrm{g})$, Clindamycin(2 $\mu \mathrm{g})$, Azithromycin(15 $\mu \mathrm{g})$, Cotrimoxazole $(1.25 / 23.75 \mu \mathrm{g})$, Gentamycin $(10 \mu \mathrm{g})$, Doxycycline $(30 \mu \mathrm{g})$, Cefoxitin $(30 \mu \mathrm{g})$. Antibiotics used for gram negative organisms were Ampicillin $(10 \mu \mathrm{g})$, Gentamycin $(10 \mu \mathrm{g})$, Cotrimoxazole(1.25/75 $\mu \mathrm{g})$, Aztreonam $(30 \mu \mathrm{g})$, Ceftazidime $(30 \mu \mathrm{g})$, Cefotaxim(30 $\mu \mathrm{g})$, Amikacin $(30 \mu \mathrm{g})$, Amoxiclavulanic $\operatorname{acid}(30 \mu \mathrm{g})$, $\operatorname{Imipenam}(10 \mu \mathrm{g})$, Piperacillin-tazobactam $\quad(100 / 10$ $\mu \mathrm{g})$.Interpretation was done as per CLSI guidelines. ${ }^{9}$

\section{Results}

A total of 156 samples were received at clinical laboratory Department of microbiology, attached to McGann teaching hospital SIMS, Shimoga from suspected cases of neonatal septicemia during the period of July 2017 and December 2017.Among 156 samples 98(62.82\%) were culture positive and $58(37.17 \%)$ were culture negative. Coagulase negative staphylococci(CoNS) was the predominant organism isolated accounting for $48.97 \%$ (48/98), followed by Klebsiella species 21.4\% (21/98), Staphylococcus aureus $10.2 \%$ (10/98), Gram negative non fermenters 9.1\% (9/98), Escherichia coli 2.04\%(2/98), Citrobacter spp 4.08\%(4/98), Pseudomonas spp 1.02\% (1/98), Enterococcus spp $1.02 \%(1 / 98)$,Streptococcus spp 1.02\%(1/98),Candida spp 1.02\%(1/98).[Graph 1]

Antibiotic susceptibility pattern of Coagulase negative staphylococci(CoNS): In our study CoNS was highly sensitive

Vancomycin(100\%),

to $\quad$ Linezolid(100\%),

Cotrimoxazole $(79.16 \%)$,

Gentamicin(75\%), Ciprofloxacin(70.83\%) and least sensitive to Clindamycin(50\%) ,Doxycycline (50\%) ,Cefoxitin(41.66\%),

Erythromycin $(33.33 \%)$. [Table 1]

\section{Discussion}

Neonatal septicemia is a clinical syndrome of bacteremia characterized by systemic signs and symptoms in first month of life. Early diagnosis and treatment can reduce mortality and morbidity. WHO estimates about 4-5million neonatal deaths every year, most of them occurring in developing country. ${ }^{(1,2,3)}$ This study was conducted to know the prevalence and antibiotic susceptibility pattern of CoNS in neonatal septicemia patients visiting McGann hospital, SIMS, Shimoga.

In this study Coagulase negative staphylococci(CoNS) was the most common organism isolated accounting for $48.97 \%$ of cases Similar study done by Shivanna et al showed $66.66 \%$ CoNS organisms isolated. ${ }^{10}$ According to study done by Jyothi et al $27.5 \%$ CoNS and $30.5 \%$ Klebsiella species were isolated. ${ }^{11}$ There is increase in isolation of Coagulase negative staphylococci(CoNS).They are skin commensals, common inhabitants of mucous membrane. Neonates acquire infection from hospital staff, hospital environment, parents when in NICU and pediatric wards. Colonisation of skin and indwelling catheters are most important sources of CoNS sepsis. ${ }^{12}$ Endemic strains are circulated because of transmission of organisms from hands of hospital staff. Increase in CoNS neonatal sepsis is attributed to immature adaptive immune response of the neonates. It is also because of lack of vernix caseosa coating of skin which normally is antimicrobial. Lack of stratum corneum, lack of virulence factors, insufficient recruitment of neutophils and biofilm formation in indwelling catheters are all responsible for CoNS pathogenesis in neonatal septicemia. ${ }^{12,13}$ Another study done by Thakur et al showed Staphylococcus aureus(40\%) followed by Coagulase negative staphylococcus(16\%) .Similarly study done by Sharma et al showed Staphylococcus aureus $(37.22 \%)$ as most common organism followed by Klebsiella pneumonia $(27.01 \%)$ where as Coagulase negative staphylococcus $(4.38 \%)$ was isolated less in number. ${ }^{15}$

In our study CoNS was highly sensitive to Linezolid (100\%), Vancomycin (100\%), Cotrimoxazole $(79.16 \%)$, Gentamicin $(75 \%)$, ciprofloxacin $(70.83 \%)$. Study done by thakur et al showed high sensitivity to Vancomycin(100\%), Clindamycin(80\%). ${ }^{14}$ Similar results showed in studies done by Jyothi et al Linezolid(91\%), tetracycline(68\%), ciprofloxacin(52\%) and Shivanna et al Linezolid(100\%) and Vancomycin $(100 \%){ }^{10,11}$ In this study high resistance was seen Cefoxitin (58.34\%), Azithromycin(66.66\%), 
erythromycin(66.66\%), Clindamycin(50\%). Similarly study by Shivanna et reported $60 \%$ resistance to Cefoxitin. ${ }^{10}$ Similarly study done by Jyothi et al showed resistance to Penicillin(90\%), cloxacillin(84\%) ,Azithromycin(67\%), erythromycin(65\%) and Thakur et al showed resistance to penicillin $(87 \%)$, amoxyclav $(66 \%)$, Cefoxitin(40\%). ${ }^{11,14}$

All these studies indicate increase in prevalence of methicillin resistant Coagulase negative staphylococci (MRCoNS) and increase in trend of multidrug resistance. MRCoNS is important nosocomial pathogen mainly in neonatal intensive care units. It is also source of resistance gene to other gram positive cocci including Staphylococcus aureus in hospital settings. Antibiotic resistance is because of various mechanism like alteration in penicillin binding proteins which leads to modification of target structures, production of betalactamases which hydrolyse penicillins, cephalosporins and carbapenems.

Preventive measures are taken against neonatal sepsis. Hand washing is most important measure to avoid CoNS infection and prevent its transfer from hospital staff to the newborn. Minimization of invasive interventions like removal of central venous catheter's promptly and reduction in the mechanical ventilation. Early entral nutrition should be promoted for enhancing infant's gastrointestinal immune response. Judicious use of antibiotics is also important preventive measure. ${ }^{13,16}$

\section{Graph 1: Distribution of organisms}

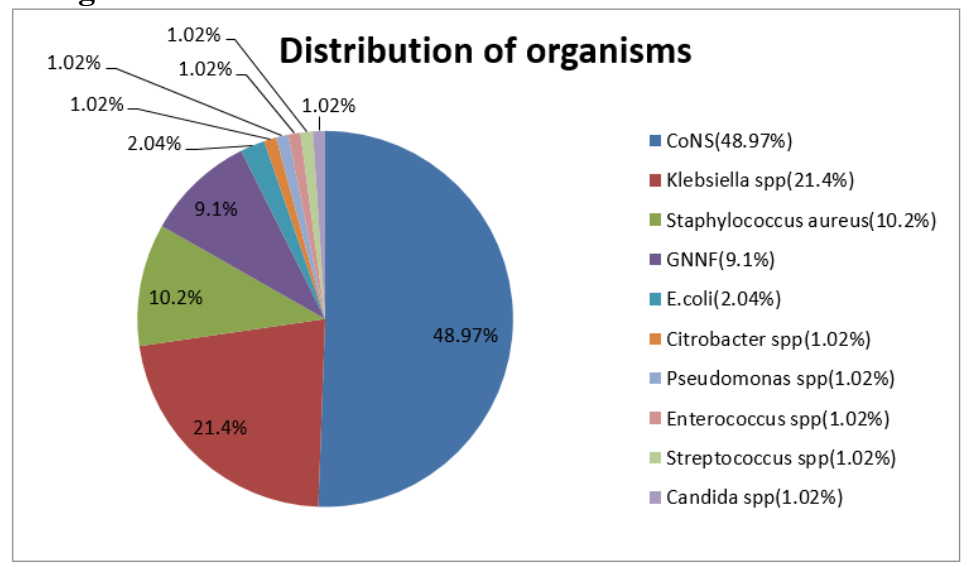

Table 2: Antibiotic susceptibility pattern of CoNS

\begin{tabular}{|l|c|}
\hline \multicolumn{1}{|c|}{ Antibiotics } & $\begin{array}{c}\text { Coagulase Negative } \\
\text { Staphylococci(CoNS)(\%) }\end{array}$ \\
\hline Linezolid & 100 \\
\hline Vancomycin & 100 \\
\hline Gentamicin & 75 \\
\hline Ciprofloxacin & 70.83 \\
\hline Doxycycline & 50 \\
\hline Azithromycin & 33.33 \\
\hline Cefoxitin & 41.66 \\
\hline Cotrimoxazole & 79.16 \\
\hline Clindamycin & 50 \\
\hline Erythromycin & 33.33 \\
\hline
\end{tabular}

\section{Conclusion}

Gram positive organisms are emerging as commonest cause of neonatal septicemia. CoNS is the commonest cause in this study. Linezolid and Vancomycin were the most sensitive drugs. High resistance was seen in Cefoxitin, Azithromycin, erythromycin and moderate resistance was seen in Gentamicin, Cotrimoxazole, Clindamycin. Most organisms showed multidrug resistance. Multidrug resistance is due to biofilm formation and indiscriminate use of antibiotics. There is also prevalence of MRCoNS. Antibiotics should not be used indiscriminately and antibiotic policy should be formulated in the hospital. Preventive measures like barrier nursing, proper hand washing and judicious use of antibiotics should be implemented.

\section{References}

1. Vrishali M, Dyaneshwari G, Arvind V. Bacteriological profile of Neonatal septicemia in a tertiary care Hospital from western India J Glob Infect Dis. 2015 AprJun; 7(2):75-7. 
2. Monjur F, Rizwan F, Asaduzzaman M, Nasrin N, Ghosh NK, Apu AS, et al. Antibiotic sensitivity pattern of causative organisms of neonatal septicemia in an urban hospital of Bangladesh. Indian J Med Sci 2010;64:265-71.

3. Nepal HP, Acharya A, Gautam R, Shresta S, Paudei R. Bacteriological profile of neonatal septicaemia cases and the antimicrobial resistance pattern in a tertiary care hospital of Central Nepal. Int J Biomed Res 2013;4:26-31.

4. Mustafa M, Ahmed SL. Bacteriological profile and antibiotic susceptibility patterns in neonatal septicaemia in view of emerging drug resistance. J Med Allied Sci 2014;4:2-8.

5. Agnihotri N, Kaistha N, Gupta V. Antimicrobial susceptibility of isolates from neonatal septicemia. Jpn J Infect Dis 2004;57:273-5.

6. Collee JG, Marr W. Culture of bacteria. In: Collee JG, Fraser AG, Marmion BP, Simmons A, editors. Mackie and McCartney Practical Medical Microbiology. 14ed. NewYork: Churchill Livingstone; 1996. p.113-29.

7. Garner JS,Jarvis WR,Emori TG,Horan TC,Hughes JM.CDC definitions for nosocomial infections, 1988.Am J Infect Control.1988;16:128-40.

8. Horan TC,Andrus M,Dudeck MA.CDC/NHSN surveillance definition of health care-associated infection and criteria for specific types of infections in the acute care setting.Am J Infect control 2008;36:309-32.

9. Clinical and laboratory institute.Performance standards for antimicrobial susceptibility testing. $27^{\text {th }}$ informational supplement.2017;M100.

10. Shivanna V, Sunkappa SR, Venkatesha D. The rising trend of coagulase-negative staphylococci in neonatal septicemia. Indian J Pathol Microbiol 2016;59:510-2.

11. P.Jyothi, Metri C.Basavaraj,and Peerapur

V.Basavraj.Bacteriological profile of neonatal septicemia and antibiotic susceptibility pattern of the isolates.J Nat Sci Biol Med.2013 Jul-Dec;4(2):306-9.

12. Jean-Baptiste N, Benjamin DK Jr., Cohen-Wolkowiez M, Fowler VG Jr., Laughon M, Clark RH, et al. Coagulase-negative staphylococcal infections in the neonatal intensive care unit. Infect Control Hosp Epidemiol 2011;32:679-86.

13. Marchant EA, Boyce GK, Sadarangani M, Lavoie PM. Neonatal sepsis due to coagulase negative staphylococci. Clin Dev Immunol.2013:586076.doi:10.1155/2013/586076.Epub 2013 May 22

14. Thakur S, Thakur K, Sood A, Chaudhary S. Bacteriological profile and antibiotic sensitivity pattern of neonatal septicaemia in a rural tertiary care hospital in North India. Indian J Med Microbiol 2016;34:67-71.

15. Chandra S, Ravi A, Hariom S, Bijay K, Deepthi S, Santokh B. Neonatal sepsis: Bacteria and their susceptibility pattern toward Antibiotics in Neonatal intensive Care Unit.J Clin Diagn Res.2013 Nov; 7(11):2511-3.

16. Kaistha N, Mehta M, Singla N, Garg R, Chander J. Neonatal septicemia isolates and resistance patterns in a tertiary care hospital of North India. J Infect Dev Ctries 2009;4:55-7. 